\title{
A Method for Solving Group Linguistic Decision-Making Problems Based on IOWA
}

\author{
Nini Duan Zheng Pei Li Qin \\ School of Mathematics \& Computer Science, Xihua University, Chengdu 610039, China
}

\begin{abstract}
In this paper, we will provide a method for solving group decision-making problems based on IOW A operators. In which, linguistic assessments of decision makers could be selected freely, i.e., let $S=$ $\left\{s_{1}, s_{2}, \cdots, s_{n}\right\}$ be basic linguistic assessments, decision maker could select his (or her) linguistic assessment from $S$, or freely give, such as, $s_{t}$, where $t \in[1, n]$. Formally, $s_{t} \in\left[s_{i}, s_{i+1}\right](i \in \mathcal{N})$ could be explained by linguistic assessment of the decision maker lied between $s_{i}$ and $s_{i+1}$. The $O W A$ pairs of IOWA operator are obtain by assessment level of experts and linguistic assessment of experts. Especially, we provide a new method to deal with 'tie' of IOW A operators. Example shows that our method is feasible.
\end{abstract}

Keywords: $O W A$ operator, IOWA operator, Group decision-making, Aggregation

\section{Introduction}

The induced aggregation operators are an interesting research topic, which are receiving increasing attention. In [1], Yager, et al introduce a class of induced ordered weighted averaging (IOWA) operators which take as their argument pairs, called $O W A$ pairs, in which one component is used to induce an ordering over the second components which are exact numerical values and then aggregated. In most voting system, the decisions are based on the condition that the selection projects are given, in this paper, we allow the decision makers give any semantic expression as long as to according with their desire. In this paper, the assessment level of decision makers based on linguistic assessment matrix are adopted as the order inducing value $u_{i}$, which together with linguistic assessments $a_{i}$ of decision makers to construct $O W A$ pair, $\left\langle u_{i}, a_{i}\right\rangle$. By aggregating all $O W A$ pairs, we can obtain the final result. In this paper, a new method is proposed to deal with 'tie'. This paper is set out as follows. In Section 2, we summarize the IOWA operators. In
Section 3, a new method to deal with 'tie' is proposed. In section 4 , we analyze the method of this paper. An example is in Section 5. In Section 6, we draw our conclusions.

\section{Preliminaries}

In [6], as noted the $O W A$ aggregation, $F_{W}\left(a_{1}, \ldots, a_{n}\right)=W^{T} B$, makes use the reordering operator $B=\operatorname{Reorder}(A)$. The ordering is based upon the value of the arguments, $b_{j}$ is the value that is the $j$ th largest of the arguments. Inspired by the work in [16], it appears that we can consider a more general policy towards the formulation of the ordered argument vector $B$. In this more general framework, we shall assume each of the argument values to be aggregated, $a_{i}$ is a component of a more complex object which we shall for our immediate purpose represent as a two-tuple $\left\langle u_{i}, a_{i}\right\rangle$ and denote as an $O W A$ pair. In this more general approach to $O W A$ aggregation, we shall order the arguments, form the vector $B$, based upon the $u_{i}$ values. In particular, our procedure for calculating the $O W A$ aggregation of these $O W A$ pairs, $F_{W}\left(\left\langle u_{1}, a_{1}\right\rangle, \ldots,\left\langle u_{n}, a_{n}\right\rangle\right)=W^{T} B_{u}$ is as follows. We form the ordered argument vector $B_{u}$ so that $b_{j}$ is the $a_{i}$ value of the $O W A$ pair having the $j$ th largest $u$ value. In discussing these $O W A$ pairs, $\left\langle u_{i}, a_{i}\right\rangle$, because of its role we shall refer to the $u_{i}$ as the order inducing variable and $a_{i}$ as the argument variable. The following simple example illustrates the approach:

Example 1 Assume we have four $O W A$ pairs $\langle 3,0\rangle,\langle 7,0.2\rangle,\langle 2,0.9\rangle,\langle 6,1\rangle$ we want to aggregate using the weighting vector $W^{T}=[0.4,0.3,0.2,0.1]$

The first step is to order the $O W A$ pairs $\left\langle u_{i}, a_{i}\right\rangle$ based on the ordering inducing variable $u_{i}$.

\section{Ordered OWA Pairs}


From this order we obtain the components of the vector $B$ by taking the ordered list of the $a_{i}$ values thus $b_{1}=0.2, b_{2}=1, b_{3}=0$ and $b_{4}=0.9$. Using this ordering, we get $F_{W}\left(\left\langle u_{i}, a_{i}\right\rangle\right)=\sum_{j=1}^{4} w_{j} b_{j}=$ $(0.4)(0.2)+(0.3)(1)+(0.2)(0)+(0.1)(0.9)=0.47$

Let us look at the properties associated with these Induced Ordered Weighted Average (IOWA) Operators, $F_{W}\left(\left\langle u_{i}, a_{i}\right\rangle\right)$. These operators are symmetric, each of the objects involved in the aggregation are treated in the same way. These operators exhibit the bounding property characteristic of mean operators, for any order inducing variable and any weighting vector

$$
\operatorname{Min}_{i}\left[a_{j}\right] \leq F_{W}\left(\left\langle u_{i}, a_{i}\right\rangle\right) \leq \operatorname{Max}_{j}\left[a_{j}\right]
$$

These IOW $A$ operators are monotonic, if $a_{i} \geq$ $\widehat{a_{i}}$ for all $i$ then $F_{W}\left(\left\langle u_{i}, a_{i}\right\rangle\right) \geq F_{W}\left(\left\langle u_{i}, \widehat{a_{i}}\right\rangle\right)$.

There are a number of ways in which the aggregation of $O W A$ pairs is different from the aggregation of $O W A$ singletons. For example, if $W$ is the Max aggregation operator, $w_{1}=1$ and $w_{j}=0$ for $j \neq 1$. In the ordinary case this returns the largest of the $a_{i}$ in the IOWA, it returns the argument value of the pair having the largest $u$ value.

An important issue that must be addressed when using these IOWA operators arises when there is a tie in the ordering operation. In the following section we will give the method to deal with the problem.

\section{The new method to deal with the 'tie'}

In [1], Yager introduce a method to manage the 'tie'. Consider aggregation of the objects $\langle 5,1\rangle$, $\langle 3,0.5\rangle,\langle 8,0.6\rangle,\langle 5,0.4\rangle$ under the weighting vector $w_{t}=[0.3,0.25,0.25,0.2]$

\section{Ordered OWA Pairs}

$$
\text { tie }\langle 5,1\rangle \quad\langle 5,0.4\rangle
$$

We see that there is a tie between $\langle 5,1\rangle$ and $\langle 5,0.4\rangle$ with respect to order inducing variable. It can be easily shown that if we brake this tie by selecting $\langle 5,1\rangle$ ahead of $\langle 5,0.4\rangle$ giving us the ordered argument vector

$$
B=\left[\begin{array}{c}
0.6 \\
1 \\
0.4 \\
0.5
\end{array}\right]
$$

We would get a different aggregated value then by selecting $\langle 5,0.4\rangle$ ahead of $\langle 5,1\rangle$, which would get the ordered argument vector

$$
B=\left[\begin{array}{c}
0.6 \\
0.4 \\
1 \\
0.5
\end{array}\right]
$$

That is $(0.4)(0.6)+(0.3)(1)+(0.2)(0.4)+$ $(0.1)(0.5) \neq(0.4)(0.6)+(0.3)(0.4)+(0.2)(1)+$ $(0.1)(0.5)$.

In [1] the policy we shall follow in the case of ties in the order inducing process is to replace the arguments of the tied $O W A$ pairs by the average of the arguments of the tied pairs in forming the $B$ vector. Thus in the preceding illustration, when forming the $B$ matrix we replace the argument component of each of $\langle 5,1\rangle$ and $\langle 5,0.4\rangle$ by their average $0.7,(1+0.4) / 2$.

This substitution gives us an ordered argument vector

$$
B=\left[\begin{array}{l}
0.6 \\
0.7 \\
0.7 \\
0.5
\end{array}\right]
$$

Following this process can be essentially shown to be equivalent to calculating the aggregated value as $W^{T} B=\frac{1}{2}\left[W^{T} B_{1}+W^{T} B_{2}\right]$. We note if $q$ items are tied, we replace these by $q$ replica's of their average.

Although Yager's method can manage the tie, but the original objects are changed. Here we introduce a new method to deal with the tie without the change of the objects.

Let $Z=\left\{\left\langle u_{i}, a_{i}\right\rangle \mid i=1,2, \cdots n\right\}$ be all $O W A$ pairs. $\left\langle u_{i}, a_{i}\right\rangle,\left\langle u_{j}, a_{j}\right\rangle$ is a tie, i.e., $u_{i}=u_{j}$

For $\left\{a_{i} \mid i=1,2, \cdots n\right\}$, the weight of $a_{i}$ in $O W A$ operator is denoted as $w_{i}=\frac{b_{i}^{\alpha}}{\sum_{i=1}^{n} b_{i}^{\alpha}}$, where $b_{i}$ is the $i$ th largest element of the collection of the aggregated objects $a_{1}, a_{2}, \ldots a_{n}, \alpha \in(-\infty, \infty)$ here we let $\alpha=0.5, a=w_{i} a_{i}+w_{j} a_{j}$, we use $\left\langle u_{i}, a\right\rangle$ instead of $\left\langle u_{i}, a_{i}\right\rangle$ and $\left\langle u_{j}, a_{j}\right\rangle$ in $\left\{\left\langle u_{i}, a_{i}\right\rangle \mid i=1,2, \cdots n\right\}$ ,i.e., $Z^{\prime}=\left\{\left\langle u_{1}, a_{1}\right\rangle, \cdots,\left\langle u_{i-1}, a_{i-1}\right\rangle,\left\langle u_{i}, a\right\rangle, \cdots\right.$, $\left.\left\langle u_{j-1}, a_{j-1}\right\rangle,\left\langle u_{j+1}, a_{j+1}\right\rangle, \cdots,\left\langle u_{n}, a_{n}\right\rangle\right\}$

with the value of $w$ we have introduced, we will have $I O W A_{w}\left\{\left\langle u_{i}, a_{i}\right\rangle \mid i=1,2, \cdots n\right\}=$ $I O W A_{w}^{\prime}\left\{\left\langle u_{1}, a_{1}\right\rangle, \cdots, \quad\left\langle u_{i-1}, \quad a_{i-1}\right\rangle,\left\langle u_{i}, \quad a\right\rangle\right.$, $\left.\cdots,\left\langle u_{j-1}, a_{j-1}\right\rangle,\left\langle u_{j+1}, a_{j+1}\right\rangle, \cdots,\left\langle u_{n}, a_{n}\right\rangle\right\}$ 
Utilize the same illustration we have the aggregation of the objects $\langle 5,1\rangle,\langle 3,0.5\rangle,\langle 8,0.6\rangle$ , $<5,0.4>$. Performing the ordering of the objects we get

Ordered OWA Pairs

$$
\text { tie }\langle 5,1\rangle\langle 5,0.4\rangle
$$

Here we divide the objects into three parts $\langle 8,0.6\rangle,(\langle 5,1\rangle,\langle 5,0.4\rangle),\langle 3,0.5\rangle$ so we can see that in the second part we can consider the IOWA operator as the $O W A$ operator. Here we need three $w$ to weight the three parts. Utilize the value of the $w$ in [5] $w_{i}=\frac{b_{i}^{\alpha}}{\sum_{i=1}^{n} b_{i}^{\alpha}}$, so we can have the $w=[1,(0.6126,0.3874), 1]$. It should be note that here we consider the $u$ as the $b_{i}$, while in the $O W A$ operator, the $b_{i}$ should be the object themselves. We consider the $0.6126 \times 1+$ $0.3874 \times 0.4=0.76756$ as the second parts of the new IOW $A$ operator, so we have the new IOW $A$ $\langle 8,0.6\rangle,\langle 5,0.76756\rangle,\langle 3,0.5\rangle$. Use the same method we get the $w=[0.41,0.32,0.26]$, so $I O W A=$ $0.6 \times 0.42+0.76756 \times 0.32+0.5 \times 0.26=0.6276$. With the same $w$, use Yager's method, we obtain the $I O W A=0.3 \times 0.6+0.25 \times 0.7+0.25 \times 0.7+$ $0.2 \times 0.5=0.63$.

We can express our new method as we divide the objects into several parts depend upon how much ties in the ordering operation. We put the object have the same $u$ together and consider them as the $O W A$ operator, aggregate the objects, we can obtain a new IOWA operator. Use the same method to obtain $w$ to aggregate the new IOWA base on the $u$, then we can get the final result. If $k(k>2)$ items are tied, we do this for $k$ times.

It should be clear that in the usual $O W A$ aggregation ties don't present a problem, the reason for this is that the ordering variable is the same as the argument variable and however we place the tied objects leads to the same result.

\section{Obtaining linguistic assess- ment and assessment level of experts}

The linguistic approach is an approximate technique, which represents qualitative aspects as linguistic values by means of linguistic variables [7][14]. Suppose that $S=\left\{s_{i} \mid i=-t, \ldots t\right\}$ is a finite and totally ordered discrete term set, where $s_{i}$ represents a possible value for a linguistic variable. For example, a set of nine terms $S$ could be $S=\left\{s_{0}=\right.$ extremely poor, $s_{1}=$ very poor, $s_{2}=$ poor, $s_{3}=$ slightly poor, $s_{4}=$ fair, $s_{5}=$ slightly good, $s_{6}=$ good, $s_{7}=$ very good, $s_{8}=$ extremely good $\}$ in which $s_{i}<s_{j}$ if $i<j$

To preserve all the given information, we extend the discrete term set $S$ to a continuous term set $S=\left\{s_{\alpha} \mid \alpha \in[-t, t]\right\}$. If $s_{\alpha} \in S$, then we call $s_{\alpha}$ an original linguistic term, otherwise, we call $s_{\alpha}$ a virtual linguistic term [15]. In general, the decision makers use the original linguistic terms to evaluate alternatives, and the virtual linguistic terms can only appear in operation [4].

In general, we can't give a fuzzy linguist information a value, although we know which semantic interval it lies. But if we can change our thinking, videlicet we don't give the fuzzy linguist information a certain semantic value, while we utilize the number axis, let the decision maker provide the certain position of the fuzzy linguist information in the number axis, through the compute of the distance we can just use it as a certain semantic to deal with.

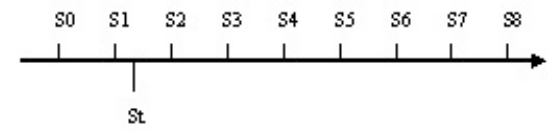

Fig. 1: The number axis

With the number axis, we let the decision maker provide the evaluation, with the computer of the distance, we can have the certain value, such as from the number axis we will know $s_{t}$ which lies between $S_{1}$ and $S_{2}$, its value is $S_{1.2}$. So we can obtain the linguist assessment matrix.

We should note that there exist different background, knowledge and so on among the decision makers, so it is difficult to ensure the value of the evaluate to consistent, so it is very important to analyze assessment level of experts. When we get the linguistic assessment matrix, we can use the method in [2] to analyze assessment level of the decision maker, we can consider the evaluation as the $u$ for the $I O W A$ operator, so we will finally deal with the whole problem.

The follows definitions is appeared in [2], with them we can get the assessment level of the decision makers.

Definition $1[6]$ suppose $\left(r_{1}, a_{1}\right),\left(r_{2}, a_{2}\right), \ldots,\left(r_{m}\right.$, 
$\left.a_{m}\right)$ is a group of evaluation of the individual decision maker which will be aggregated, so LWD operator be defined as

$$
\left(r_{E}, a_{E}\right)=L W D\left(\left(r_{1}, a_{1}\right), \ldots,\left(r_{m}, a_{m}\right)\right)
$$

thereinto, $a_{E}$ is the evaluation of the group, $r_{E}$ is the important degree of the group evaluations. They can be obtained as follow:

$$
\begin{gathered}
a_{E}=\max \left\{\min \left(r_{1}, a_{1}\right), \ldots, \min \left(r_{m}, a_{m}\right)\right\} \\
r_{E}=\Phi_{L}\left(r_{1}, r_{2}, \ldots, r_{m}\right)
\end{gathered}
$$

In [16] the author give the definition and property of declination between two linguist.

Definition $2 a_{i}^{k}$ and $a_{i}^{l}$ is the lingual evaluation of export $e_{k}$ and $e_{i}$, thereinto, $a_{i}^{k}=s_{u}, a_{i}^{l}=s_{v}$, $s_{u}, s_{v} \in S$, then $\rho\left(a_{i}^{k}, a_{i}^{l}\right)$ or $\rho_{k l}^{i}$ is the coherence index of alternative aggregation $x_{i}$ by export $e_{k}$ and $e_{l}$

$$
\rho\left(s_{u}, s_{v}\right)=1-\frac{|u-v|}{T}
$$

Definition $3 X^{k}=\left[a_{1}^{k}, a_{2}^{k}, \ldots a_{n}^{k}\right]$ is the project sorting representation vector, $\forall k \in J$, thereinto, $a_{i}^{k}$ is the language evaluation of $x_{i}$ by export $e_{k}$, $\rho\left(a_{i}^{k}, a_{i}^{l}\right)$ or $\rho_{k l}^{i}$ is the coherence index of alternative aggregation $x_{i}$ by export $e_{k}$ and $e_{l}, C_{i}$ is the coherence vector of $x_{i}$ by the group of exports, $\mu_{i}$ is the coherence index of $x_{i}$ by the group of exports.

$$
\begin{array}{r}
C_{i}=\left[\rho_{12}^{i} \rho_{13}^{i} \ldots \rho_{1 m}^{i} \rho_{23}^{i} \rho_{24}^{i} \ldots \rho_{2 m}^{i} \rho_{m-1, m}^{i}\right] \\
\mu_{i}=\phi\left(\rho_{12}^{i}, \ldots, \rho_{1 m}^{i}, \rho_{23}^{i}, \rho_{24}^{i}, \ldots, \rho_{2 m}^{i}, \rho_{m-1, m}^{i}\right)
\end{array}
$$

Definition 4 Suppose $\rho\left(a_{i}^{k}, a_{i}^{l}\right)$ or $\rho_{k l}^{i}$ is the coherence index of alternative aggregation $x_{i}$ by export $e_{k}$ and $e_{l}$ thereinto, $\rho_{k l}^{i}=\rho_{l k}^{i}, \forall l, k \in J$, then $C^{k l}$ is the coherence vector of $x_{i}$ by export $e_{k}$ and $e_{l}, \mu^{k l}$ is the coherence index of $x_{i}$ by export $e_{k}$ and $e_{l}$

$$
\begin{aligned}
& C^{k l}=\left[\rho_{k l}^{1} \rho_{k l}^{2} \ldots \rho_{k l}^{n}\right] \\
& \mu^{k l}=\phi\left(\rho_{k l}^{1}, \ldots, \rho_{k l}^{n}\right)
\end{aligned}
$$

Definition 5 suppose $\mu^{k l}$ is the coherence index by export $e_{k}$ and $e_{l}$, then $C^{k}$ is the coherence vector of by the export $e_{k}$ and the group of exports, $\mu^{k}$ is the coherence index by the export $e_{k}$ and the group of exports.

$$
\begin{gathered}
C^{k}=\left[\mu^{k 1} \mu^{k 2} \mu^{k(k-1)} \mu^{k(k+1)} \ldots \mu^{k m}\right] \\
\mu^{k}=\phi\left(\mu^{k 1}, \ldots, \mu^{k(k-1)}, \mu^{k(k+1)}, \ldots, \mu^{k m}\right)
\end{gathered}
$$

\section{Example}

Let us suppose a vote problem, which want the customer give the evaluation about the home appliances market. Suppose alternative aggregation $X=x_{1}, x_{2}, x_{3}, x_{4}$, index aggregation $P=$ $p_{1}, p_{2}, p_{3}, p_{4}$, and there are five experts in this problem. There is a panel with four possible alternatives.

(1) $x_{1}$ is the television

(2) $x_{2}$ is the refrigeratory

(3) $x_{3}$ is the roller washing machine

(4) $x_{4}$ is the air-condition

There are four attributes as follow:

(1) The price

(2) The quality

(3) The after service

(4) The exterior

The four possible alternatives $x_{i}(i=1,2,3,4)$ are evaluated using the linguistic term set

$S=\left\{s_{0}=\right.$ extremely poor, $s_{1}=$ very poor, $s_{2}=$ poor, $s_{3}=$ slightly poor, $s_{4}=$ fair, $s_{5}=$ slightly good, $s_{6}=$ good, $s_{7}=$ very good, $s_{8}=$ extremely good \}

Use the method we have introduce in part 3, utilize the number axis and the method in [2], we can get the value of the evaluation as following

$$
B=\left[\begin{array}{c}
0.6 \\
1 \\
0.4 \\
0.5
\end{array}\right]
$$

$$
\begin{aligned}
R^{1} & =\left[s_{6.6}, s_{7}, s_{6.5}, s_{6}\right] \\
R^{2} & =\left[s_{6}, s_{7}, s_{5.5}, s_{5.5}\right] \\
R^{3} & =\left[s_{6}, s_{6}, s_{5.5}, s_{5.5}\right] \\
R^{4} & =\left[s_{8}, s_{7.5}, s_{7}, s_{7}\right] \\
R^{5} & =\left[s_{6}, s_{6}, s_{5.5}, s_{7.5}\right] \\
A_{1} & =\left(\begin{array}{cccc}
s_{1.2} & s_{1.7} & s_{1.6} & s_{2.1} \\
s_{6.5} & s_{7} & s_{6} & s_{6} \\
s_{5} & s_{6.2} & s_{5.8} & s_{5.5} \\
s_{6} & s_{5.4} & s_{6.3} & s_{4.9}
\end{array}\right) \\
A_{2} & =\left(\begin{array}{cccc}
s_{3.9} & s_{4.1} & s_{4.5} & s_{4.7} \\
s_{6} & s_{6.2} & s_{5.5} & s_{5.4} \\
s_{5.2} & s_{5} & s_{4.6} & s_{4.4} \\
s_{4.9} & s_{5} & s_{4.6} & s_{4}
\end{array}\right) \\
A_{3} & =\left(\begin{array}{cccc}
s_{4.4} & s_{4.9} & s_{5} & s_{5.5} \\
s_{5.9} & s_{5.7} & s_{5.5} & s_{4} \\
s_{4.4} & s_{4.7} & s_{3.8} & s_{3.9} \\
s_{4} & s_{3.3} & s_{2.9} & s_{4.4}
\end{array}\right) \\
A_{4}= & \left(\begin{array}{cccc}
s_{0.9} & s_{1} & s_{0.8} & s_{1.1} \\
s_{7.5} & s_{7.1} & s_{6.6} & s_{6} \\
s_{6.9} & s_{7.3} & s_{7} & s_{6.6} \\
s_{7} & s_{5.6} & s_{6.7} & s_{5.5}
\end{array}\right)
\end{aligned}
$$


$A_{5}=\left(\begin{array}{cccc}s_{5.2} & s_{5.7} & s_{5.5} & s_{7.5} \\ s_{5.5} & s_{5.7} & s_{5} & s_{3.9} \\ s_{4} & s_{4.2} & s_{3.7} & s_{3.5} \\ s_{3.7} & s_{2} & s_{3.5} & s_{4}\end{array}\right)$

Utilize (1)-(3), we can obtain the projects' sequencing of every customer

$a_{i}^{1}=\left(s_{2.1}, s_{7}, s_{6.2}, s_{6.3}\right)$

$a_{i}^{2}=\left(s_{4.7}, s_{6.2}, s_{5.2}, s_{5}\right)$

$a_{i}^{3}=\left(s_{5.5}, s_{5.9}, s_{4.7}, s_{4.4}\right)$

$a_{i}^{4}=\left(s_{1.1}, s_{7.5}, s_{7.3}, s_{7}\right)$

$a_{i}^{5}=\left(s_{5.7}, s_{7}, s_{4.2}, s_{4}\right)$

We use the proportional fuzzy quantify "the most", with the formulation $4-10$, we have $\mu^{12}=$ $\phi\left(\rho_{12}^{1}, \rho_{12}^{2}, \rho_{12}^{3}, \rho_{12}^{4}\right)=(0.675,0.9,0.875,0,8375)=$ 0.88125 . With the same method we will have $\mu^{13}=$ $0.8275, \mu^{14}=0.885, \mu^{15}=0.78125, \mu^{23}=0.94625$, $\mu^{24}=0.77875, \mu^{25}=0.9, \mu^{34}=0.725, \mu^{35}=$ $0.95375, \mu^{45}=0.67875$. $\mu^{1}=\phi\left(\mu^{11}, \mu^{12}, \mu^{13}\right.$, $\left.\mu^{14}, \mu^{15}\right)=(1,0.88125,0.8275,0.885,0.78125)=$ 0.86125 , with the same method we get $\mu^{2}=0.89$, $\mu^{3}=0.87925, \mu^{4}=0.84575, \mu^{5}=0.833$

The $\mu^{i}$ is the induced variable in the new $I O W A$ operator. Utilize the value of the $w[5]$, $w_{i}=\frac{b_{i}^{\alpha}}{\sum_{i=1}^{n} b_{i}^{\alpha}}$ we have the

$w^{T}=[0.2033,0.202,0.1999,0.1981,0.1967]$

Now we have five group $O W A$ pairs.

\section{Ordered OWA Pairs}

$$
\begin{gathered}
\left\langle 0.89, s_{4.7}\right\rangle \\
\left\langle 0.87925, s_{5.5}\right\rangle \\
\left\langle 0.86125, s_{2.1}\right\rangle \\
\left\langle 0.84575, s_{1.1}\right\rangle \\
\left\langle 0.833, s_{7.5}\right\rangle
\end{gathered}
$$

Ordered OWA Pairs

$$
\begin{gathered}
\left\langle 0.89, s_{6.2}\right\rangle \\
\left\langle 0.87925, s_{5.9}\right\rangle \\
\left\langle 0.86125, s_{7}\right\rangle \\
\left\langle 0.84575, s_{7.5}\right\rangle \\
\left\langle 0.833, s_{5.7}\right\rangle
\end{gathered}
$$

Ordered OWA Pairs

$$
\begin{gathered}
\left\langle 0.89, s_{5.2}\right\rangle \\
\left\langle 0.87925, s_{4.7}\right\rangle \\
\left\langle 0.86125, s_{6.2}\right\rangle \\
\left\langle 0.84575, s_{7.3}\right\rangle
\end{gathered}
$$

$$
\begin{gathered}
\left\langle 0.833, s_{4.2}\right\rangle \\
\text { Ordered } O W A \text { Pairs } \\
\left\langle 0.89, s_{5}\right\rangle \\
\left\langle 0.87925, s_{4.4}\right\rangle \\
\left\langle 0.86125, s_{6.3}\right\rangle \\
\left\langle 0.84575, s_{7}\right\rangle \\
\left\langle 0.833, s_{4}\right\rangle
\end{gathered}
$$

so the final aggregation result is

$I O W A_{w}^{1}=\left(\left\langle 0.89, s_{4.7}\right\rangle,\left\langle 0.87925, s_{5.5}\right\rangle,\langle 0.86125\right.$ ,$\left.\left.s_{2.1}\right\rangle,\left\langle 0.84575, s_{1.1}\right\rangle,\left\langle 0.833, s_{7.5}\right\rangle\right)=0.2033 \times 4.7+$ $0.202 \times 5.5+0.1999 \times 2.1+0.1981 \times 1.1+0.1967 \times$ $7.5=4.17942$

$$
I O W A_{w}^{2}=\quad\left(\left\langle 0.89, \quad s_{6.2}\right\rangle, \quad\langle 0.87925,\right.
$$
$\left.s_{5.9}\right\rangle, \quad\left\langle 0.86125, \quad s_{7}\right\rangle, \quad\left\langle 0.84575, \quad s_{7.5}\right\rangle, \quad\langle 0.833$, $\left.\left.s_{5.7}\right\rangle\right)=0.2033 \times 6.2+0.202 \times 5.9+0.1999 \times 7+$ $0.1981 \times 7.5+0.1967 \times 5.7=6.4585$

$I O W A_{w}^{3}=\left(\left\langle 0.89, s_{5.2}\right\rangle,\left\langle 0.87925, s_{4.7}\right\rangle,\langle 0.86125\right.$, $\left.\left.s_{6.2}\right\rangle,\left\langle 0.84575, s_{7.3}\right\rangle,\left\langle 0.833, s_{4.2}\right\rangle\right)=0.2033 \times 5.2+$ $0.202 \times 4.7+0.1999 \times 6.2+0.1981 \times 7.3+0.1967 \times$ $4.2=5.51821$

$I O W A_{w}^{4}=\left(\left\langle 0.89, s_{5}\right\rangle,\left\langle 0.87925, s_{4.4}\right\rangle,\langle 0.86125\right.$, $\left.\left.s_{6.3}\right\rangle,\left\langle 0.84575, s_{7}\right\rangle,\left\langle 0.833, s_{4}\right\rangle\right)=0.2033 \times 5+$ $0.202 \times 4.4+0.1999 \times 2.1+0.1981 \times 7+0.1967 \times 4=$ 4.49859

Rank all the alternatives $x_{i}(i=1,2,3,4)$ in accordance we have $x_{2}>x_{3}>x_{4}>x_{1}$, thus the best alternative is $x_{2}$

\section{Conclusion}

In this paper, we provide a method for solving group decision-making problems based on IOW $A$ operators. In which, linguistic assessments of decision makers could be selected freely. The $O W A$ pairs of $I O W A$ operator are obtain by assessment level of experts and linguistic assessment of experts. Especially, we provide a new method to deal with 'tie' of $I O W A$ operators. Example shows that our method is feasible.

\section{Acknowledgement}

This work is supported by the Excellent Young Foundation of Sichuan Province (Grant No.06ZQ026-037), the National Natural Science 
Foundation of China (60474022), the Special Research Funding to Doctoral Subject of Higher Education Institutes in China (Grant No.20060613007) and a Project Supported by Scientific Research Fund of Sichuan Provincial Education Department(Grant No.2005A121, 2006A084).

\section{References}

[1] R. R. Yager, D. P. Filev, Operations for granular computing:mixing words and numbers, Proceedings of the FUZZ-IEEE World Congress on Computational Intelligence, Anchorage, pp. 123-128, 1998.

[2] X. Chen , Z. P. Fan,Method Based on Linguistic Assessment Matrix to Analyze Assessment Level of Experts. Journal of Northeastern University(Natural Science), 27(5):587-590, 2006.

[3] R. R. Yager, On ordered weighted averaging aggregation operators in multi-criteria decision making. IEEE Transactions on Systems, Man, and Cybernetics, 18:183-190, 1988.

[4] Z. S. Xu, Induced uncertain linguistic OWA operators applied to group decision making. Information Fusion, 7:231-238, 2006.

[5] Z. S. Xu, An overview of methods for determining OWA weights. International Journal Of Intelligent Systems, 18:953-969, 2003.

[6] F. Herreera, E. Herrera-Viedma, Aggregation operators for linguistic weighted information. IEEE Transactions on Systems, Man, and Cybernetics, Part A: Systems and Humans, 27(5):646-656, 1997.

[7] L. A. Zadeh, The concept of a linguistic variable and its application to approximate reasoning. Part 1, 2, 3. Information Sciences, 8:199-249, 301-357, 1975, 9:43-80, 1976.

[8] L. A. Zadeh, A computational approach to fuzzy quantifiers in natural languages. Comput. Math. Appl, 9:149-184, 1983.

[9] M. T. Lamata, A model of decision with linguistic knowledge. Mathware and Soft Computing, 3:253-263, 1994.

[10] L. A. Zadeh, J. Kacprzyk, Computing with Words in Information.Intelligent Systems-Part 1: Foundations; Part 2: Applications, vol. I, Physica-Verlag, Heidelberg, 1999.

[11] F.Herrera, E.Herrera-Viedma, Linguistic decision analysis: steps for solving decision problems under linguistic information. Fuzzy Sets and Systems, 115:67-82, 2000.

[12] F. Herrera, E. Herrera-Viedma, J. L. Verdegay, A model of consensus in group decision making under linguistic assessments. Fuzzy Sets and Systems, 78:73-87,1996.

[13] F. Herrera, L. Martinez, A 2-tuple fuzzy linguistic representation model for computing with words. IEEE Transactions on Fuzzy Systems, 8:746-752, 2000.

[14] F. Herrera, L. Martinez, A model based on linguistic 2-tuples for dealing with multigranular hierarchical linguistic contexts in multiexpert decision-making. IEEE Transactions on Systems, Man, and Cybernetics-Part B, 31:227234, 2001.

[15] Z. S. Xu, A method based on linguistic aggregation operators for group decision making with linguistic preference relations. Information $\mathrm{Sci}$ ences, in press.

[16] Z. S. Xu, Deviation measures of linguistic preference relations in group decision making. The International Journal of Management Science,10(1): 1-6, 2004. 